\title{
Foreword
}

Our intention is to present studies that explore the deep connections between theories of integral education and the practices of new integrations in education, with an emphasis on drama education in general, and Integral DramaBased Pedagogy in particular. We are indebted to an international group of scholars, teachers, and practitioners who have contributed studies that draw upon their knowledge of theories of integral education from various times and cultures as well as their personal experiences in developing practices that exemplify and inspire new integrations.

This collection of essays is especially relevant considering the current global evolution of primary, secondary, and higher education. This evolution is encouraging teachers to expand traditional disciplinary boundaries to transdisciplinary approaches that integrate multiple ways of knowing and recognize the fullness of being human.

We use 'Integral Education' as a general designation that includes a variety of educational models that draw upon the works of many innovative thinkers, past and present, who have proposed theories and developed practices that exemplify integrative models of education. We maintain that these models reflect a set of common characteristics as we have referenced in our "Introduction" and as others have in their essays. We believe that Integral Education is not a limited vision shaped by a particular person, theory, or practice; but rather, it is an expansive approach with a common array of characteristics. Thus, Integral Education is our shorthand description of multiple models that have in common the integration of many areas of learning; include multiple ways of knowing; and recognize the intellectual, physical, and spiritual dimensions of being human.

While we have arranged our collection according to two principal themes of theory and practice, we recognize that both themes are integrated into each essay.

We are grateful to Prof. Michael Peters, the journal editor, for providing us with an opportunity to co-edit this special issue of the Beijing International Review of Education and the forthcoming expanded volume; and to Ms. Hejia 
Wang, the journal manager, for her thoughtful preparation for publication of this special issue and the forthcoming volume.

\section{Liwen MA}

Associate Professor, Institute of Educational Psychology and School

Counseling, Faculty of Education, Beijing Normal University, Beijing, China liwenma@126.com

Joseph L. SUBBIONDO

Trustee, California Institute for Human Science Encinitas, CA, USA jsubbiondo@gmail.com 